\title{
Symmetry breaking induced by random fluctuations for Bose-Einstein condensates in a double-well trap
}

\author{
J. Garnier \\ Laboratoire de Statistique et Probabilités, Université Paul Sabatier, \\ 118 Route de Narbonne, 31062 Toulouse Cedex 4, France, \\ Tel. (33) 5615562 20, Fax. (33) 5615560 89, Email: garnier@cict.fr \\ F. Kh. Abdullaev \\ Physical-Technical Institute of the Uzbek Academy of Sciences, \\ G. Mavlyanov str. 2-b, 700084, Tashkent, Uzbekistan
}

(Dated: October 30, 2018)

\begin{abstract}
This paper is devoted to the study of the dynamics of two weakly-coupled Bose-Einstein condensates confined in a double-well trap and perturbed by random external forces. Energy diffusion due to random forcing allows the system to visit symmetry-breaking states when the number of atoms exceeds a threshold value. The energy distribution evolves to a stationary distribution which depends on the initial state of the condensate only through the total number of atoms. This loss of memory of the initial conditions allows a simple and complete description of the stationary dynamics of the condensate which randomly visits symmetric and symmetry-breaking states.

PACS numbers: 03.75.Kk, 03.75.Lm, 05.40.Ca
\end{abstract}

\section{INTRODUCTION}

The experimental achievements of Bose-Einstein condensates (BECs) with various dilute trapped alkali-metal atom gases have opened new possibilities to investigate macroscopic quantum effects [1]. Indeed it is well known that phase coherence plays a crucial role in macroscopic quantum systems, and different systems have been addressed: Josephson effect in superconductors [2], weakly linked superfluid He-B reservoirs [3], two coupled BECs in a double well trap [4], and coupled condensates in different hyperfine levels [5]. In this paper we shall address the quantum coherent tunneling between two BECs trapped in a double-well potential, and we shall focus our attention to the influence of random external fluctuations. In superconductors the main origin of external noise is thermal or quantum fluctuations. Mathematically the problem can be reduced to the pendulum equation (sine-Gordon equation) with external fluctuations $\phi_{t t}+\sin (\phi)+\gamma \phi_{t}=f(t)[\underline{6}]$ and the energy diffusion of the system can be studied. In particular Stratonovich has studied this problem in the strong damping case $\gamma \phi_{t}+\sin (\phi)=f(t)$ []].

In BECs new types of problems appear due to the existence of the trap potential. Coherent phase phenomena in coupled BECs are described by a system of ordinary differential equations for the imbalance in atomic population $z$ and relative phase $\phi$. This system can be considered as the equation for the nonrigid pendulum. The pendulum case recovered if the imbalance is small $z^{2} \ll 1$. Nonrigidity leads to new phenomena, and we can distinguish two regimes in the atomic imbalance evolution. The first regime is the macroscopic quantum tunneling (MQT), when the atomic population is periodically exchanged between wells with $\langle z\rangle=0$, which is analogous to the Josephson effect. The second regime is the macroscopic quantum localization (MQL) phenomena, when the mean field nonlinearity excesses some critical value, the mean value of the imbalance becomes nonzero $\langle z\rangle \neq 0$, which corresponds to the localization of the atomic population in one of the wells in the form of small-amplitude oscillations around the bottom of the well.

It is therefore interesting to investigate the influence of fluctuations on the evolution of the system in these states. In particular we would like to pay a particular attention to the possible switching between these states. This problem has a general character and it is important for the theory of nonlinear directional couplers in nonlinear optics $[8,[9]$ and nonlinear dimers [10]. Periodic variations of the parameters of the trap have already been considered, and resonant phenomena have been exhibited for the weakly-coupled BEC [11] and for the strongly overlapped BEC [12]. The influence of a periodic time-varying atomic scattering length was addressed in [13]. Dynamical tunneling between regions of regular motion was shown to be involved by strong periodic modulation of the tunnel coupling between the two modes [14]. Finally macroscopic quantum chaos driven by a time-periodic trap asymmetry was predicted in 15. In this work we shall study the influence of random fluctuations on the BEC dynamics trapped in a double-well trap. We shall address the case of fluctuating zero point energies so that the energy difference $\Delta E(t)$ is a zero-mean random process. Such variations are induced by small oscillations in the barrier laser position 16, 17]. Indeed, the barrier is generated by a Gaussian laser sheet focused near the center of the harmonic trap and small oscillations of the barrier-laser position introduce the fluctuations of the zero-point energies. 


\section{BOSE-EINSTEIN CONDENSATES IN A DOUBLE-WELL TRAP}

The problem of BECs in a double-well time-dependent trap is described by the Gross-Pitaevskii (GP) equation

$$
i \hbar \Psi_{t}=-\frac{\hbar^{2}}{2 m} \Delta \Psi+V_{t r}(r, t) \Psi+g|\Psi|^{2} \Psi,
$$

where $V_{t r}$ is the double-well potential, $g=4 \pi \hbar^{2} a_{s} / m$, and $a_{s}$ is the atomic scattering length. For weakly overlapped condensates (high barrier or well separated wells) we can use the two-modes decomposition

$$
\Psi=\phi_{1}(t) \Phi_{1}(r)+\psi_{2}(t) \Phi_{2}(r)
$$

where $\psi_{1,2}$ are complex time-dependent amplitudes of condensates in wells and $\Phi_{1,2}$ are approximate ground state solutions of GP equation in first and second wells respectively. Substituting this solution into (1), multiplying the equation by $\overline{\Phi_{1,2}}$ and integrating over the spatial variable we obtain the system of equations for the two modes $\psi_{1}(t), \psi_{2}(t)[18,19]$

$$
\begin{aligned}
& i \hbar \frac{\partial \psi_{1}}{\partial t}=\left[E_{1}(t)+\alpha_{1}\left|\psi_{1}\right|^{2}\right] \psi_{1}-K \psi_{2}, \\
& i \hbar \frac{\partial \psi_{2}}{\partial t}=\left[E_{2}(t)+\alpha_{2}\left|\psi_{2}\right|^{2}\right] \psi_{2}-K \psi_{1},
\end{aligned}
$$

where $E_{1,2}$ are the zero point energies, $K$ is the coupling constant between the two modes, and $\alpha_{1,2}$ are the mean field nonlinearities for both modes. Here we take into account a small oscillation in the laser-barrier position so that $\Delta E=E_{1}-E_{2}$ is time-varying [20]. We write $\psi_{j}=\sqrt{N_{j}} \exp \left(i \theta_{j}\right) . N_{T}=N_{1}+N_{2}$ is the (constant) total number of atoms. The fractional population imbalance

$$
z(t)=\frac{N_{1}(t)-N_{2}(t)}{N_{T}}
$$

and the relative phase

$$
\phi(t)=\theta_{2}(t)-\theta_{1}(t)
$$

satisfy

$$
\begin{aligned}
& z_{t}=-\sqrt{1-z^{2}} \sin (\phi)-\eta \phi_{t}, \\
& \phi_{t}=-\Delta E(t)+\Lambda z+\frac{z}{\sqrt{1-z^{2}}} \cos (\phi),
\end{aligned}
$$

where we have rescaled to a dimensionless time $t 2 K / \hbar \rightarrow t$ and we have introduced new variables

$$
\begin{aligned}
& \Delta E(t)=\frac{E_{1}(t)-E_{2}(t)}{2 K}+\frac{\alpha_{1}-\alpha_{2}}{4 K} N_{T}, \\
& \Lambda=\frac{\alpha N_{T}}{2 K}, \quad \alpha=\frac{\alpha_{1}+\alpha_{2}}{2} .
\end{aligned}
$$

Note that we have included the damping term $-\eta \phi_{t}$ in Eq. (7) that takes into account a non-coherent dissipative current of normal-state atoms, proportional to the chemical potential difference 11].

\section{HAMILTONIAN STRUCTURE OF THE UNPERTURBED SYSTEM}

The Hamiltonian of the unperturbed system $(\Delta E=0, \eta=0)$ is

$$
E=\frac{\Lambda z^{2}}{2}-\sqrt{1-z^{2}} \cos (\phi) .
$$

$E$ can take any value between $E_{\min }=-1$ and $E_{\max }=\frac{1}{2}\left(\Lambda+\frac{1}{\Lambda}\right)$. It is an integral of motion. The orbits of the motion are closed, corresponding to periodic oscillations. In order to explicit the periodic structure of the variables $z$ and $\phi$, 
we introduce the action-angle variables. The orbits are completely determined by the energy $E$ imposed by the initial conditions. The period is denoted by $\mathcal{T}(E)$, the motion is described by

$$
\begin{aligned}
& z(t)=\mathcal{Z}(E, \theta(t)), \\
& \cos (\phi(t))=\mathcal{C}(E, \theta(t)), \\
& \sin (\phi(t))=\mathcal{S}(E, \theta(t)),
\end{aligned}
$$

where $\mathcal{Z}, \mathcal{C}$, and $\mathcal{S}$ are smooth functions, periodic with respect to $\theta$ with period $2 \pi$, the angle satisfies

$$
\frac{d \theta}{d t}=\frac{2 \pi}{\mathcal{T}(E)}
$$

and the action is defined by

$$
\mathcal{I}(E)=\frac{1}{2 \pi} \int_{-1}^{E} \mathcal{T}(x) d x
$$

The analysis is standard but quite cumbersome, so we only list the final results that can be written in terms of elliptic functions. We need to introduce a series of parameters.

$$
\begin{aligned}
& \kappa(E)=\frac{\Lambda E-1}{2}, \quad \zeta(E)=\frac{\Lambda^{2}-1}{4}-\kappa(E) \\
& M(E)=\frac{1}{2}\left(1+\frac{\kappa}{\sqrt{\zeta}}\right), \quad z_{2}(E)=\frac{2}{\Lambda} \sqrt{\kappa+\sqrt{\zeta}}
\end{aligned}
$$

Three cases can be distinguished.

1. If $E \in[-1,1)$, then $M<1$, the period is

$$
\mathcal{T}(E)=\frac{8 \sqrt{M}}{\Lambda z_{2}} K(M),
$$

where $K$ is the complete elliptic function [22],

$$
\begin{aligned}
& \mathcal{Z}(E, \theta)=z_{2} \mathrm{cn}\left(\frac{2 K(M) \theta}{\pi}, M\right) \\
& \mathcal{C}(E, \theta)=\frac{\Lambda \mathcal{Z}^{2}-2 E}{2 \sqrt{1-\mathcal{Z}^{2}}}, \\
& \mathcal{S}(E, \theta)=\frac{\Lambda z_{2}^{2}}{2 \sqrt{M} \sqrt{1-\mathcal{Z}^{2}}} \operatorname{sn} \operatorname{dn}\left(\frac{2 K(M) \theta}{\pi}, M\right) .
\end{aligned}
$$

$\mathrm{cn}, \mathrm{sn}$, and dn are tabulated Jacobian functions [22]. Note that $\mathcal{Z}$ is an even function with respect to $\theta$. These solutions preserve the $z$-symmetry in the sense that they correspond to eigenfunctions of the Gross-Pitaevskii equation that are either odd or even functions. The stationary ground state has energy -1 and it is given by $z \equiv 0$ and $\phi \equiv 0$. For $E \in(-1,1)$, we have $\langle z\rangle=0$ and the atomic population is periodically exchanged between the two modes. This is the MQT regime.

2. If $E \in\left(1, E_{\max }\right)$, then $M>1$, the period is

$$
\mathcal{T}(E)=\frac{4}{\Lambda z_{2}} K\left(\frac{1}{M}\right) .
$$

The three periodic functions $\mathcal{Z}, \mathcal{C}$, and $\mathcal{S}$ are given by

$$
\begin{aligned}
& \mathcal{Z}(E, \theta)= \pm z_{2} \operatorname{dn}\left(K\left(\frac{1}{M}\right) \frac{\theta}{\pi}, \frac{1}{M}\right) \\
& \mathcal{C}(E, \theta)=\frac{\Lambda \mathcal{Z}^{2}-2 E}{2 \sqrt{1-\mathcal{Z}^{2}}}, \\
& \mathcal{S}(E, \theta)=\frac{\Lambda z_{2}^{2}}{2 M \sqrt{1-\mathcal{Z}^{2}}} \operatorname{sn} \operatorname{cn}\left(K\left(\frac{1}{M}\right) \frac{\theta}{\pi}, \frac{1}{M}\right) .
\end{aligned}
$$


a)

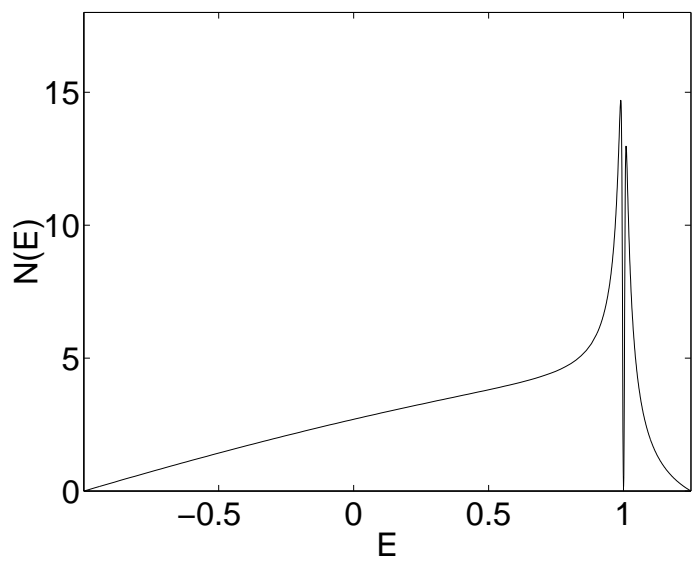

b)

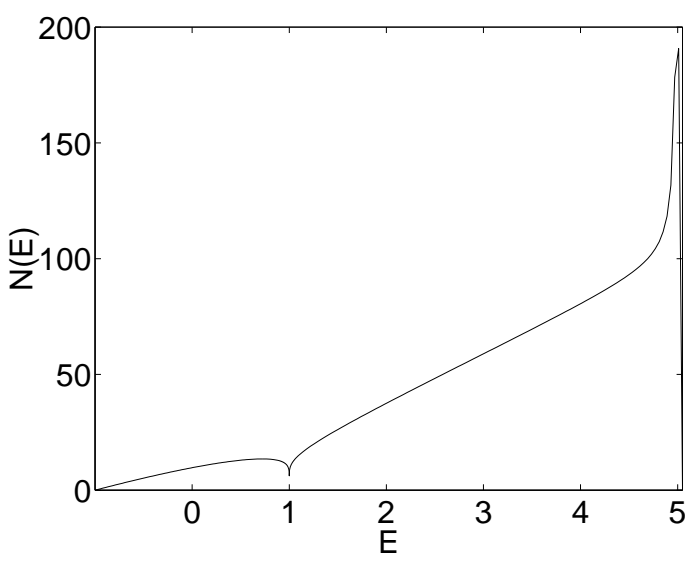

FIG. 1: Damping term $E \mapsto \mathcal{N}(E)$ for $\Lambda=2$ (picture a) and $\Lambda=10$ (picture b).

Note that $\mathcal{Z}$ is either always positive, or always negative-valued. These solutions correspond to eigenfunctions that break the $z$-symmetry. In particular there exist two stationary solutions with the maximal energy $E_{\max }$ that are $z \equiv \pm \sqrt{1-1 / \Lambda^{2}}, \phi \equiv \pi$. Their existences result from the nonlinear interatomic interaction and it is possible only when the number of atoms is large enough so that nonlinear coefficient $\Lambda>1$. If $E \in\left(1, E_{\max }\right)$ the population imbalance periodically oscillates around a non-zero average value $\langle z\rangle \neq 0$. This is the MQL regime.

3. If $E=1$, then $M=1$, the motion is given, in this special case, by the non-oscillatory hyperbolic secant

$$
z(t)=z_{2} \operatorname{sech}\left(\sqrt{\Lambda-1}\left(t+t_{0}\right)\right),
$$

with $z_{2}=2 \sqrt{\Lambda-1} / \Lambda$ and $t_{0}=(1 / \sqrt{\Lambda-1}) \operatorname{argch}\left(z_{2} / z_{0}\right)$.

\section{EFFECTIVE DYNAMICS}

\section{A. Damping}

In presence of a small damping an adiabatic approach is possible which yields that the energy decays as

$$
\frac{d E}{d t}=-\eta \mathcal{N}(E)
$$

where

$$
\mathcal{N}(E)=\frac{1}{2 \pi} \int_{0}^{2 \pi}\left(\Lambda \mathcal{Z}+\frac{\mathcal{Z}}{\sqrt{1-\mathcal{Z}^{2}}} \mathcal{C}\right)^{2}(E, \theta) d \theta .
$$

$\mathcal{N}(E)$ vanishes at $E=-1, E=1$, and $E=E_{\max }$. It takes large values just above and below $E=\Lambda / 2$. In Figure 1 we plot the function $E \mapsto \mathcal{N}(E)$ for two different values of $\Lambda$. Expansions of $\mathcal{N}$ are also presented in the Appendix.

Damping is not able by itself to break the symmetry as it involves an energy decay, while symmetry breaking occurs when the energy goes from a value below the separatrix 1 to a value above the separatrix. If the system starts from one of the two breaking states with the energy $E_{\max }$ then it stays exactly at this state as $\mathcal{N}\left(E_{\max }\right)=0$. However, a linear stability analysis shows that these states are not stable. More exactly, if the initial state has energy $E_{\max }-e$ with $e \ll 1$, then it stays close to this state during a time of the order of $\sim\left[\Lambda\left(\Lambda^{2}-1\right) e\right]^{-1}$ because $\mathcal{N}\left(E_{\max }-e\right) \simeq \Lambda\left(\Lambda^{2}-1\right) e$; after this time it quits the breaking state and converges to the ground state $E_{\min }$. This behavior is described in Figure 2 Note the remarkable agreement between the results from numerical simulations of the system of equations (78) with $\Delta E=0$ and the effective solution given by Eq. (28). 
a)

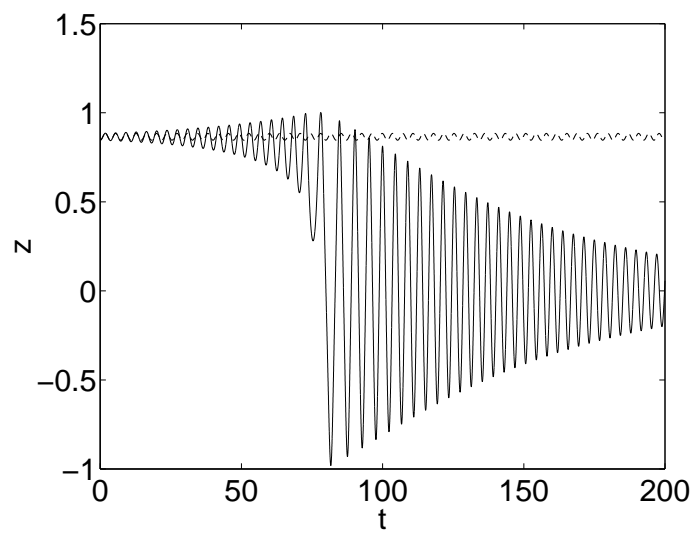

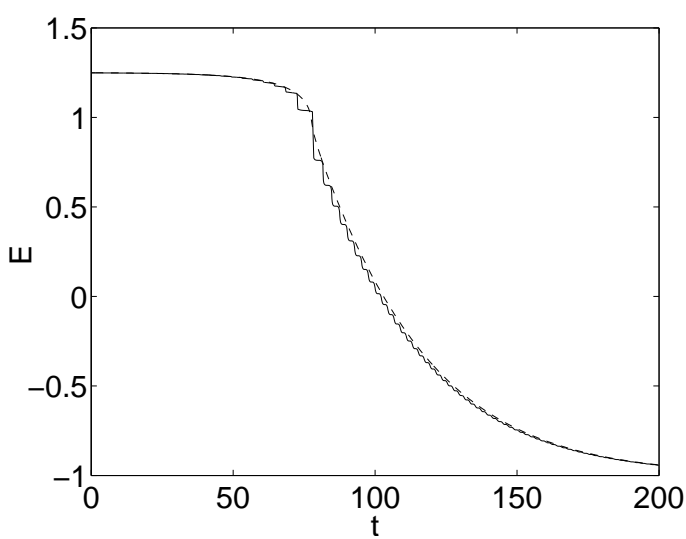

FIG. 2: Picture a: Evolution of the population imbalance $z$ in absence (dashed line) and in presence (solid line) of a small damping $\eta=10^{-2}$. Here $\Lambda=2$ and the initial state is $z_{0}=\sqrt{1-1 / \Lambda^{2}}-0.02 \simeq 0.846$ and $\phi_{0}=\pi$. Picture b: Evolution of the energy $E(t)=\Lambda z^{2}(t) / 2-\sqrt{1-z^{2}(t)} \cos (\phi(t))$ according to the numerical resolution of the system (7:8) (solid line) and according to the effective equation (28) (dashed line).

\section{B. Random fluctuations}

Let us consider a random perturbation of the system that can be written in the form

$$
\begin{aligned}
& z_{t}=-\partial_{\phi} H, \\
& \phi_{t}=\partial_{z} H,
\end{aligned}
$$

where $H=H_{0}(z, \phi)+m(t) V(z, \cos (\phi), \sin (\phi)), H_{0}$ is the unperturbed Hamiltonian (11), and $m(t) V$ is the timedependent perturbation. $m$ is assumed to be a stationary, zero-mean, random process. We think in particular at a time-dependent $\Delta E(t)$ in Eq. (7) so that $m(t)=\Delta E(t)$ and $V=-z$.

In presence of perturbations, the motion of $(z, \phi)$ is not purely oscillatory, because the energy and the action are slowly varying in time. We adopt the action-angle formalism, because it allows us to separate the fast scale of the locally periodic motion and the slow scale of the evolution of the action. The motion is governed by the system

$$
\begin{aligned}
& I_{t}=-m(t) h_{\theta}(I, \theta), \\
& \theta_{t}=\omega(I)+m(t) h_{I}(I, \theta),
\end{aligned}
$$

where $\omega(I)=\frac{2 \pi}{\mathcal{T} \circ \mathcal{E}(I)}, I \mapsto \mathcal{E}(I)$ is the inverse function of $E \mapsto \mathcal{I}(E)$, and $h(I, \theta)=V(\mathcal{Z}(I, \theta), \mathcal{C}(I, \theta), \mathcal{S}(I, \theta))$. Using standard diffusion-approximation theory [21], we get that the action $I$ behaves like a diffusion Markov process with the infinitesimal generator

$$
\mathcal{L}_{I}=\frac{1}{2} \frac{\partial}{\partial I}\left[A(I) \frac{\partial}{\partial I}\right]
$$

The diffusion coefficient is

$$
A(I)=\frac{1}{\pi} \int_{0}^{2 \pi} \int_{0}^{\infty} h_{\theta}(I, \theta) h_{\theta}(I, \theta+\omega(I) t)\langle m(0) m(t)\rangle d t d \theta,
$$

where the brackets stand for a statistical averaging. This means in particular that the probability density function of $I(t)$ satisfies the Fokker-Planck equation $\partial_{t} p=\mathcal{L}_{I}^{*} p, p(t=0, I)=\delta\left(I-I_{0}\right)$, where $I_{0}$ is the initial action at time 0 and $\mathcal{L}_{I}^{*}$ is the adjoint operator of $\mathcal{L}_{I}$, which is equal to $\mathcal{L}_{I}$ in our configuration as $\mathcal{L}_{I}$ is self-adjoint.

\section{Energy diffusion}

The results of the two previous subsections can be combined to address the case of system (78) with a white noise model for $\Delta E$ and a damping $\eta>0$. We get that the energy $E$ of the system is a diffusion process with the 
a)

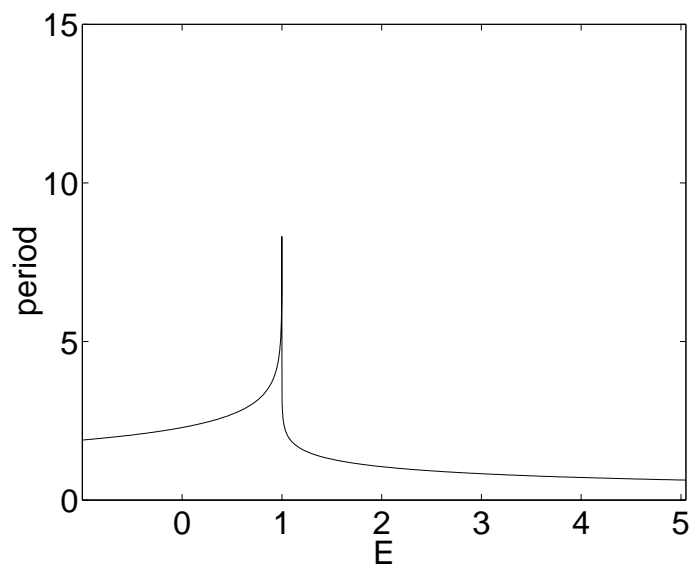

b)

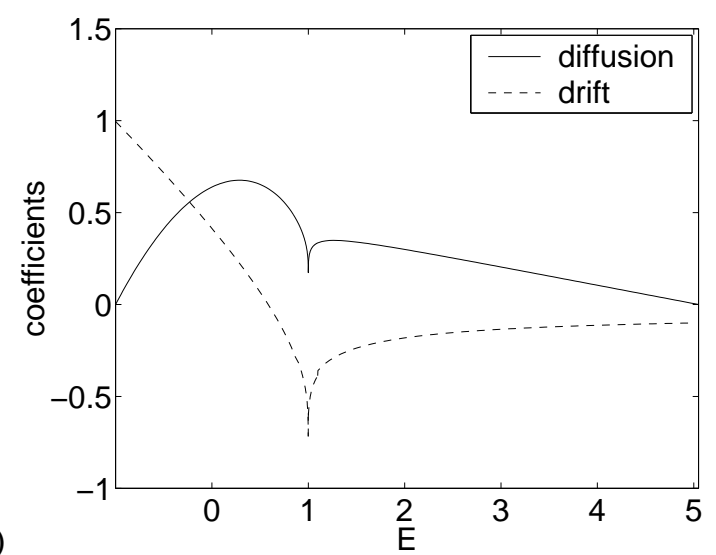

FIG. 3: Period $\mathcal{T}(E)$, drift and diffusion coefficients for $\Lambda=10$.

infinitesimal generator

$$
\mathcal{L}_{E}=\frac{2 \pi^{2} \alpha}{\mathcal{T}(E)} \frac{\partial}{\partial E}\left[\frac{\mathcal{B}(E)}{\mathcal{T}(E)} \frac{\partial}{\partial E}\right]-\eta \mathcal{N}(E) \frac{\partial}{\partial E}
$$

where

$$
\begin{gathered}
\mathcal{B}(E)=\frac{1}{2 \pi} \int_{0}^{2 \pi} \mathcal{Z}_{\theta}(E, \theta)^{2} d \theta, \\
\alpha=\int_{0}^{\infty}\langle\Delta E(0) \Delta E(t)\rangle d t .
\end{gathered}
$$

The infinitesimal generator is not self-adjoint. The drift of the energy is $d(E)=\alpha 2 \pi^{2} \partial_{E}[\mathcal{B}(E) / \mathcal{T}(E)] / \mathcal{T}(E)-\eta \mathcal{N}(E)$ and the diffusion coefficient is $\sigma(E)=\alpha 2 \pi^{2} \mathcal{B}(E) / \mathcal{T}^{2}(E)$. They are plotted in Figure 3 in the case $\eta=0$. This result allows us to compute all relevant quantities, in particular the stationary energy distribution. Indeed the diffusion process $E(t)$ is ergodic, and the statistical distribution of the energy becomes independent of the initial state for large time $t$. It converges to a stationary distribution that can be computed explicitly as the solution of the elliptic equation $\mathcal{L}_{E}^{*} p=0$.

\section{ENERGY DISTRIBUTION}

The stationary energy distribution for the system is determined by the resolution of the equation $\mathcal{L}_{E}^{*} p=0$ that is

$$
p(E)=C \mathcal{T}(E) \exp \left(-\frac{\eta}{\alpha} \int_{-1}^{E} \frac{\mathcal{T}^{2} \mathcal{N}}{2 \pi^{2} \mathcal{B}}(x) d x\right),
$$

where the normalization constant $C$ is chosen so that $\int_{-1}^{E_{\max }} p(E) d E=1$. This distribution depends only on $\Lambda$ and the ratio $\eta / \alpha$. A first important quantity is the average energy

$$
\langle E\rangle=\int_{-1}^{E_{\max }} E p(E) d E .
$$

It is plotted in Figure 4 as a function of $\Lambda$ for different damping rates, which shows in particular that the average energy grows linearly with $\Lambda$ when $\eta=0$, according to $E \simeq \Lambda / 6$, but possesses a maximum if $\eta>0$. Another important quantity is the proportion of time spent by the system in breaking states

$$
R_{b}=\int_{1}^{E_{\max }} p(E) d E
$$

which is zero for $\Lambda \leq 1$ and becomes positive for $\Lambda>1 . R_{b}$ is plotted in Figure 4 which shows that, for a given value of $\eta / \alpha$, there exists a critical value for the nonlinear coefficient $\Lambda$ such that the proportion of time spent by the system in breaking states is maximal. We can examine theoretically several particular cases. 
a)

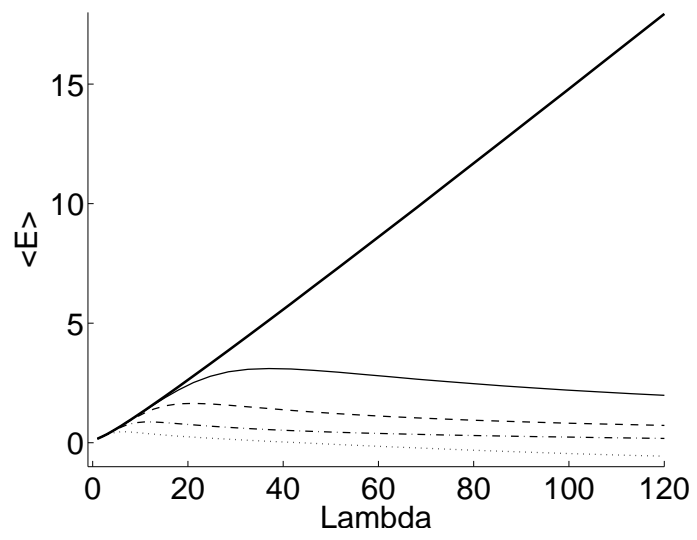

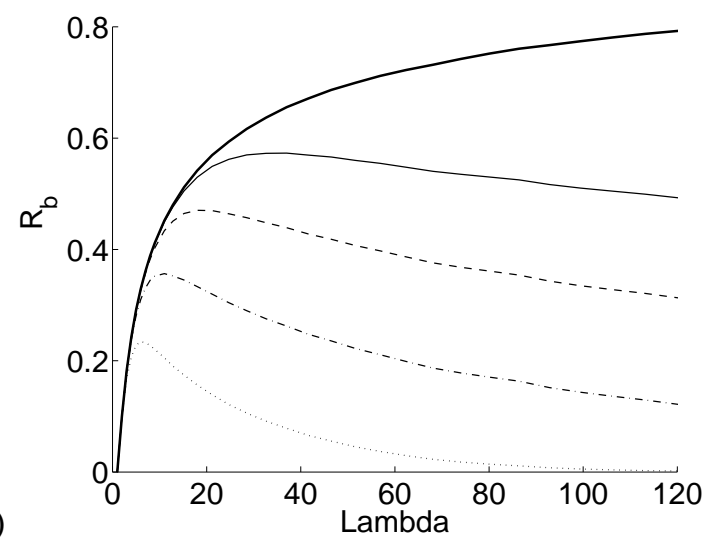

FIG. 4: Picture a: Average energy as a function of $\Lambda$ for different damping rates. Picture b: Proportion of time spent by the system in breaking states $R_{b}$ as a function of $\Lambda$ for different damping rates: $\eta / \alpha=0$ (thick solid line), $\eta / \alpha=10^{-5}$ (thin solid line), $\eta / \alpha=10^{-4}$ (dashed line), $\eta / \alpha=10^{-3}$ (dash-dotted line), $\eta / \alpha=10^{-2}$ (dotted line).

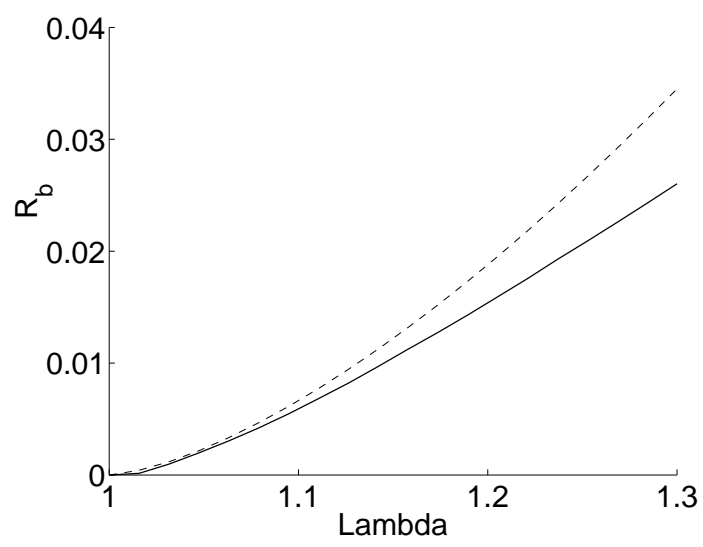

FIG. 5: Proportion of time spent by the system in breaking states $R_{b}$ as a function of $\Lambda$. The numerical integration of Eq. (41) is plotted in solid line, the approximate formula $R_{b} \simeq 0.21 \lambda^{3 / 2}$ is plotted in dashed lines.

\section{A. Absence of damping}

In absence of damping $\eta=0$, the energy distribution does not depend on the diffusion rate and it is given by

$$
p(E)=\frac{\mathcal{T}(E)}{\int_{-1}^{E_{\max }} \mathcal{T}(x) d x} .
$$

Let us first address the case where the number of atoms is just above the critical number that allows the existence of symmetry-breaking states. If $\Lambda=1+\lambda, 0<\lambda \ll 1$, then we get by using the expansions described in Appendix A 4 that

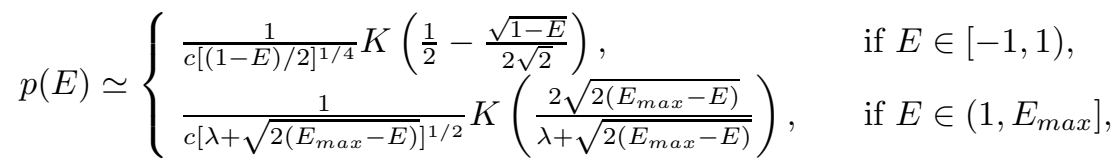

with $c \simeq 4.44$ and the proportion of time spent by the system in breaking states is $R_{b} \simeq 0.21 \lambda^{3 / 2}$. A comparison with the numerical integration of (41) shows that the approximate formula $R_{b} \simeq 0.21 \lambda^{3 / 2}$ is valid for $0 \leq \lambda \leq 0.2$ (see Figure 5). Thus, for a small number of atoms, it is not likely to observe the MQL regime.

Let us now consider the case of a large number of atoms. If $\Lambda \gg 1$, then we get by using the expansions described 
in Appendix $\mathrm{A3}$ that

$$
p(E) \simeq \begin{cases}\frac{2}{\pi \sqrt{\Lambda}} K\left(\frac{1+E}{2}\right), & \text { if } E \in[-1,1) \\ \frac{1}{\sqrt{2 \Lambda(E-1)}}, & \text { if } E \in\left(1, E_{\max }\right]\end{cases}
$$

and the proportion of time spent by the system in breaking states is $R_{b} \simeq 1-2.55 \Lambda^{-1 / 2}$. We can see in Figure 4b that this approximate formula is valid for $\Lambda \geq 40$. Thus $R_{b}$ is close to one which means that it is very likely to observe the MQT regime. We can also compute the transition time from one breaking state to the other one. This computation is based on a well-known result of stochastic analysis which claims that $\mu_{E_{0}, E_{1}}(E)$, the mean time to exit the interval $\left[E_{0}, E_{1}\right]$ starting from the energy $E$, satisfies $\mathcal{L}_{E} \mu=-1$ with the boundary conditions $\mu\left(E_{0}\right)=\mu\left(E_{1}\right)=0$. The result is that the transition time is of order $\Lambda^{2} / \alpha$. The computation of the transition time is similar to the one performed to obtain the solution to the Kramers' exit problem which is concerned with noise activated escape from a potential well [23]. As a result we can predict that, for large $\Lambda$, the system randomly goes from one breaking state to the other one according to a Markovian dynamics with an average period of $\Lambda^{2} / \alpha$.

\section{B. Small damping}

In presence of damping Eq. (39) shows that the stationary energy distribution is shifted toward the low energy region. To illustrate this phenomenon, we can consider and analyze the case $\Lambda \gg 1$ and $\eta / \alpha \ll 1 / \Lambda$. Using the expansions described in Appendix A3 the energy distribution can be written as

$$
p(E) \simeq \begin{cases}\frac{2 C_{\eta}}{\pi \sqrt{\Lambda}} K\left(\frac{1+E}{2}\right), & \text { if } E \in[-1,1), \\ \frac{C_{\eta}}{\sqrt{2 \Lambda(E-1)}} \exp \left(-2 \frac{\Lambda \eta}{\alpha} E^{2}\right), & \text { if } E \in\left(1, E_{\text {max }}\right],\end{cases}
$$

with

$$
C_{\eta}=\left[\int_{0}^{1 / 2} \frac{1}{\sqrt{2 x}} \exp \left(-\frac{\Lambda^{3} \eta}{\alpha} x^{2}\right) d x\right]^{-1} \simeq \begin{cases}1 & \text { if } \eta / \alpha \ll 1 / \Lambda^{3} \\ \frac{2 \sqrt{2}}{\Gamma(1 / 4)} \frac{\eta^{1 / 4} \Lambda^{3 / 4}}{\alpha^{1 / 4}} & \text { if } 1 / \Lambda^{3} \ll \eta / \alpha \ll 1 / \Lambda\end{cases}
$$

As a result, the proportion of time spent by the system in breaking states is $R_{b} \simeq 1-2.55 C_{\eta} \Lambda^{-1 / 2}$ which decays with $\eta$. It is less and less likely to observe the MQT regime as the damping becomes larger.

\section{Strong damping}

Assume that $\eta / \alpha \gg 1 /(1+\Lambda)$. Using the expansions derived in Subsection A1 the diffusion operator can be written as

$$
\mathcal{L}_{E}=\alpha \frac{\partial}{\partial E}(1+E) \frac{\partial}{\partial E}-\eta(1+\Lambda)(1+E) \frac{\partial}{\partial E} .
$$

The stationary energy distribution is

$$
p(E)=\frac{1}{E_{c}} \exp \left(-\frac{1+E}{E_{c}}\right) \mathbf{1}_{\left[-1, E_{\max }\right]}(E)
$$

where $E_{c}=\alpha /[(1+\Lambda) \eta]$. The average energy is $-1+E_{c}$. The proportion of time spent in breaking states is exponentially small $R_{b} \simeq \exp \left(-2 / E_{c}\right)$.

\section{Numerical experiments}

In this section we compare our theoretical predictions with numerical simulations of Eqs. (78). The random fluctuations of $\Delta E(t)$ are modeled by a stepwise constant process

$$
\Delta E(t)=\sigma \sum_{j} X_{j} \mathbf{1}_{\left[j t_{c},(j+1) t_{c}\right)}(t),
$$


a)

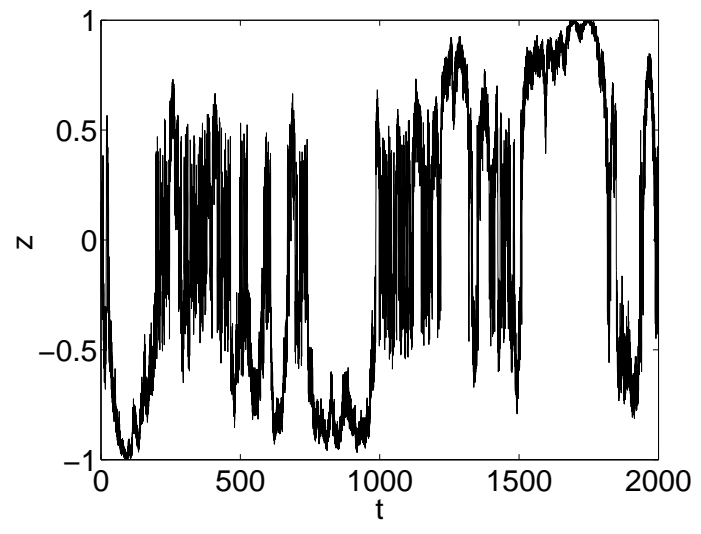

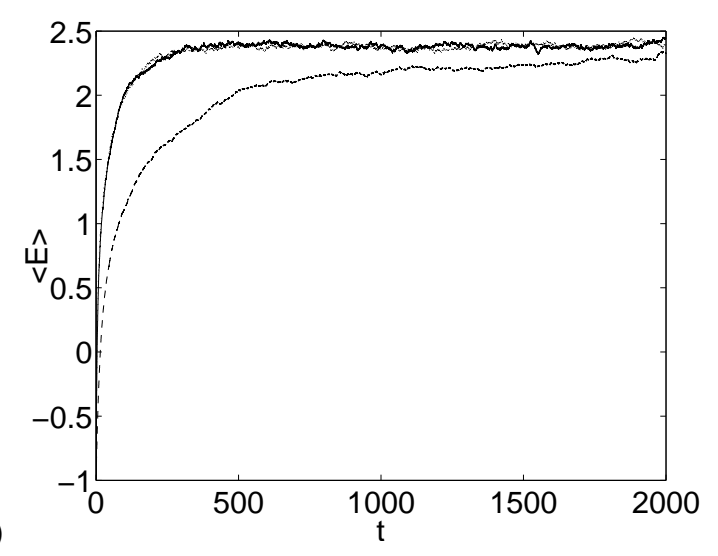

FIG. 6: Picture a: A typical $z$-trajectory for the set of parameters $\left(\sigma, t_{c}\right)=(4,0.05)$. Picture b: Average energy versus time for three different configurations: $\left(\sigma, t_{c}\right)=(4,0.05)$ (solid line), $\left(\sigma, t_{c}\right)=(3,0.1)$ (dotted line), and $\left(\sigma, t_{c}\right)=(2,0.05)($ dashed line).

where the $X_{j}$ are independent and identically distributed random variables with uniform distribution over $(-\sqrt{3}, \sqrt{3})$ and $t_{c}$ is the coherence time. The coefficient $\alpha$ is then given by

$$
\alpha=\frac{\sigma^{2} t_{c}}{2} .
$$

Damping is absent $\eta=0$ and the parameter $\Lambda=15$. Three series of simulations are performed with the parameters $\left(\sigma, t_{c}\right)=(4,0.05),\left(\sigma, t_{c}\right)=(3,0.1)$, and $\left(\sigma, t_{c}\right)=(2,0.05)$. Note that the first two configurations give almost the same value for the effective parameter $\alpha \simeq 0.4$. We have carried 1000 simulations for each configuration. The initial conditions are $z_{0}=0, \phi_{0}=0$, but we have checked that the initial conditions play no role in the long-time dynamics.

In Figure [6 a we plot a typical $z$-trajectory for the configuration $\left(\sigma, t_{c}\right)=(4,0.05)$. As predicted by the theory, noise induces an energy diffusion which makes the system visit symmetry-breaking states. More quantitatively, the system spends around half-time is symmetry-breaking states as predicted by Figure $4 \mathrm{p}$, and the life-time of a symmetrybreaking state is of the order of $\Lambda^{2} / \alpha \simeq$ a few hundreds.

In figure 6 6 we plot the energy of the system for the three configurations. The energy is averaged over the 1000 simulations. We can observe that the behavior is the same for the first two configurations, which is in agreement with the fact that both configurations possess the same effective parameter $\alpha \simeq 0.4$. We can also compare the asymptotic value of the average energy with the theoretical one $\langle E\rangle \simeq \Lambda / 6=2.5$. Note that the time necessary to reach the asymptotic value is of the order of $\Lambda^{2} / \alpha \simeq 500$. The configuration $\left(\sigma, t_{c}\right)=(2,0.05)$ corresponds to a smaller value of $\alpha=0.1$. As a result the time necessary to reach the asymptotic energy value is longer, but the asymptotic value itself is the same as for the other configurations as it depends only on $\Lambda$.

\section{CONCLUSION}

In this paper we have considered the dynamics of two weakly-coupled BECs. We have investigated the influence of small random oscillations of the barrier-laser position which induce fluctuations of the zero-point energies of the double-well trap. We have shown that random noise induces an energy diffusion which allows the system to visit symmetry-breaking states when the number of atoms (proportional to $\Lambda$ ) exceeds a threshold value. We have shown that the energy distribution evolves to a stationary distribution which depends on the initial state of the BEC only through the total number of atoms, and not through the initial imbalance or phase difference. We have computed the time necessary for the loss of memory of the initial conditions and the establishment of the stationary dynamics. Then we have described the stationary dynamics of the BEC which visits symmetric and symmetry-breaking states according to a random Markovian dynamics. We have investigated the role of damping in this process. In particular we have shown that, for a given damping, there exists a critical value for the number of atoms where the proportion of time spent by the BEC in symmetry-breaking states (i.e. in a macroscopic quantum localization state) is maximal. 


\section{APPENDIX A: EXPANSIONS}

\section{1. $E$ close to $E_{\text {min }}$}

The minimal energy is $E_{\text {min }}=-1$. We can expand all quantities when $E=E_{\text {min }}+e, e \geq 0$. We get in particular $M \simeq \frac{\Lambda^{2}}{2(1+\Lambda)^{2}} e, z_{2} \simeq \frac{\sqrt{2}}{\sqrt{1+\Lambda}} \sqrt{e}$,

$$
\begin{aligned}
& \mathcal{T}(E)=\frac{2 \pi}{\sqrt{1+\Lambda}}, \\
& \mathcal{Z}(E, \theta)=\frac{\sqrt{2}}{\sqrt{1+\Lambda}} \sqrt{e} \cos (\theta), \\
& \mathcal{C}(E, \theta)=1-e \sin (\theta)^{2},
\end{aligned}
$$

so that

$$
\begin{aligned}
& \mathcal{N}(E)=(1+\Lambda) e \\
& \mathcal{B}(E)=\frac{2 e}{1+\Lambda}
\end{aligned}
$$

\section{2. $E$ close to $E_{\max }$}

Here we assume that $\Lambda>1$ so that $E_{\max }>1$. We can expand all quantities when $E=E_{\max }-e, e \geq 0$. We get in particular $M \simeq \frac{\sqrt{2}\left(\Lambda^{2}-1\right)}{8 \sqrt{\Lambda e}}, z_{2} \simeq \frac{\sqrt{\Lambda^{2}-1}}{\Lambda}+\frac{\sqrt{2 e}}{\sqrt{\Lambda\left(\Lambda^{2}-1\right)}}$,

$$
\begin{aligned}
& \mathcal{T}(E)=\frac{2 \pi}{\sqrt{\Lambda^{2}-1}}, \\
& \mathcal{Z}(E, \theta)=\frac{\sqrt{\Lambda^{2}-1}}{\Lambda}+\frac{\sqrt{2 e}}{\sqrt{\Lambda\left(\Lambda^{2}-1\right)}} \cos (\theta), \\
& \mathcal{C}(E, \theta)=-1+\Lambda e \sin ^{2}(\theta),
\end{aligned}
$$

so that

$$
\begin{aligned}
& \mathcal{N}(E)=\Lambda\left(\Lambda^{2}-1\right) e \\
& \mathcal{B}(E)=\frac{e}{\Lambda\left(\Lambda^{2}-1\right)}
\end{aligned}
$$

\section{Large $\Lambda$}

Let us assume that $\Lambda \gg 1$. If $E \in[-1,1)$, then $M \simeq(1+E) / 2<1$,

$$
\begin{aligned}
& \mathcal{T}(E)=\frac{4}{\sqrt{\Lambda}} K\left(\frac{1+E}{2}\right), \\
& \mathcal{Z}(E, \theta)=\frac{\sqrt{2}}{\sqrt{\Lambda}} \sqrt{1+E} \operatorname{cn}\left(\frac{2 K\left(\frac{1+E}{2}\right) \theta}{\pi}, \frac{1+E}{2}\right) .
\end{aligned}
$$

If $E \in\left(1, E_{\max }\right]$, then by setting $E=\Lambda e, e \in(0,1 / 2]$, we have

$$
\begin{aligned}
& \mathcal{T}(E)=\frac{\sqrt{2} \pi}{\Lambda \sqrt{e}} \\
& \mathcal{Z}(E, \theta)=\sqrt{2 e}\left[1-\frac{\sqrt{1-2 e}}{\Lambda e} \sin ^{2}\left(\frac{\theta}{2}\right)\right],
\end{aligned}
$$


so that

$$
\begin{aligned}
\mathcal{N}(E) & =2 \Lambda^{2} e \\
\mathcal{B}(E) & =\frac{1-2 e}{2 \Lambda^{2} e}
\end{aligned}
$$

\section{4. $\Lambda$ just above 1}

This case is interesting in that it is the regime where symmetry-breaking appear. Let us assume $\Lambda=1+\lambda$, $0<\lambda \ll 1$. If $E \in[-1,1)$, then $M \simeq \frac{1}{2}\left(1-\frac{\sqrt{1-E}}{\sqrt{2}}\right)$ and

$$
\mathcal{T}(E)=\frac{2 \sqrt{2}}{E^{\prime 1 / 4}} K\left(\frac{1}{2}\left(1-\sqrt{E^{\prime}}\right)\right), \quad E^{\prime}=(1-E) / 2 .
$$

Note that $E_{\max }=1+\lambda^{2} / 2+O\left(\lambda^{3}\right)$. If $E=1+\frac{\lambda^{2}}{2} e, e \in[0,1]$, then $M \simeq \frac{1}{2}\left(1+\frac{1}{\sqrt{1-e}}\right)$ and

$$
\mathcal{T}(E)=\frac{2 \sqrt{2}}{\sqrt{1+\sqrt{e^{\prime}} \sqrt{\lambda}}} K\left(\frac{2 \sqrt{e^{\prime}}}{1+\sqrt{e^{\prime}}}\right), \quad e^{\prime}=1-e
$$

[1] F. Dalfovo, S. Giorgini, L. P. Pitaevskii, and S. Stringary, Rev. Mod. Phys. 71, 463 (1999).

[2] A. Barone and G. Paterno, Physics and Applications of the Josephson Effect (Wiley, New York, 1982).

[3] O. Avenel and E. Varoquaux, Phys. Rev. Lett. 55, 2704 (1985); S.V. Pereverzev et al. Nature (London) 388,449 (1997).

[4] A. Smerzi, S. Fantoni, S. Giovanazzi, and S. R. Shenoy, Phys. Rev. Lett. 79, 4950 (1997).

[5] J. Williams, R. Walser, J. Cooper, E. Cornell, and M. Holland, Phys. Rev. A 59, R31 (1999).

[6] K. K. Likharev, Dynamics of Josephson junctions and circuits (Gordon and Breach, New York, 1986).

[7] R. L. Stratonovich, Topics in the theory of random noise (Gordon and Breach, New York, 1963).

[8] F. Kh. Abdullaev, S. A. Darmanyan, and P. K. Khabibullaev, Optical Solitons (Springer-Verlag, Heidelberg, 1993).

[9] Y. Kivshar and G. Agrawal, Optical solitons: From fibers to photonic crystals (Academic Press, San Diego, 2003)

[10] A. C. Scott, Phys. Rep. 217, 1 (1992).

[11] F. Kh. Abdullaev and R. A. Kraenkel, Phys. Rev. A 62, 023613 (2000).

[12] P. V. Elyutin and A. N. Rogovenko, Phys. Rev. E 63, 026610 (2001).

[13] F. Kh. Abdullaev and R. A. Kraenkel, Phys. Lett. A 272, 395 (2000).

[14] G. L. Salmond, C. A. Holmes, and G. J. Milburn, Phys. Rev. A 65, 033623 (2002).

[15] W. Hai, C. Lee, G. Chong, and L. Shi, Phys. Rev. E 66, 026202 (2002).

16] N. Tsukada, M. Gotoda, Y. Nomura, and T. Isu, Phys. Rev. A 59, 3862 (1999).

[17] S. Giovanazzi, A. Smerzi, and S. Fantoni, Phys. Rev. Lett. 84, 4521 (2000).

[18] S. Raghavan, A. Smerzi, S. Fantoni, and S. R. Shenoy, Phys. Rev. A 59, 620 (1999).

19] I. Marino, S. Raghavan, S. Fantoni, S. R. Shenoy, and A. Smerzi, Phys. Rev. A 60, 487 (1999).

[20] A. Smerzi and S. Raghavan, Phys. Rev. A 61, 063601 (2000).

[21] G. C. Papanicolaou, D. Stroock, and S. R. S. Varadhan, Martingale approach to some limit theorems, Papers from the Duke Turbulence Conference (Duke Univ., Durham, N.C., 1976), Paper No. 6, Duke Univ., Durham, N.C., 1977, pp. ii+120 pp. Duke Univ. Math. Ser., Vol. III.

[22] M. Abramowitz and I. Stegun, Handbook of mathematical functions (Dover Publications, New-York, 1965).

[23] P. Hänggi, P. Talkner, and M. Borkovec, Rev. Mod. Phys. 62, 251 (1990). 